\title{
Universiteit
}

Leiden

The Netherlands

\section{Mechanism of polymer drag reduction using a low-dimensional model} Roy, A.; Morozov, A.; Saarloos, W. van; Larson, R.G.

\section{Citation}

Roy, A., Morozov, A., Saarloos, W. van, \& Larson, R. G. (2006). Mechanism of polymer drag reduction using a low-dimensional model. Physical Review Letters, 97(23), 234501. doi:10.1103/PhysRevLett.97.234501

Version: $\quad$ Not Applicable (or Unknown)

License: $\quad$ Leiden University Non-exclusive license

Downloaded from: https://hdl.handle.net/1887/66542

Note: To cite this publication please use the final published version (if applicable). 


\title{
Mechanism of Polymer Drag Reduction Using a Low-Dimensional Model
}

\author{
Anshuman Roy, ${ }^{1}$ Alexander Morozov, ${ }^{2}$ Wim van Saarloos, ${ }^{2}$ and Ronald G. Larson ${ }^{1, *}$ \\ ${ }^{1}$ Department of Chemical Engineering, University of Michigan, Ann Arbor, Michigan 48109, USA \\ ${ }^{2}$ Instituut-Lorentz, University of Leiden, Postbus 9506, 2300 RA Leiden, The Netherlands
}

(Received 22 September 2006; published 6 December 2006)

\begin{abstract}
Using a retarded-motion expansion to describe the polymer stress, we derive a low-dimensional model to understand the effects of polymer elasticity on the self-sustaining process that maintains the coherent wavy streamwise vortical structures underlying wall-bounded turbulence. Our analysis shows that at small Weissenberg numbers, Wi, elasticity enhances the coherent structures. At higher Wi, however, polymer stresses suppress the streamwise vortices (rolls) by calming down the instability of the streaks that regenerates the rolls. We show that this behavior can be attributed to the nonmonotonic dependence of the biaxial extensional viscosity on $\mathrm{Wi}$, and identify it as the key rheological property controlling drag reduction.
\end{abstract}

PACS numbers: $83.60 . Y z, 47.27 . \mathrm{Cn}, 83.10 . \mathrm{Gr}, 83.80 . \mathrm{Rs}$

It has been known since the 1940's that the addition of parts per million amounts of polymer molecules substantially reduces turbulent drag in wall-bounded parallel shear flows, such as turbulent pipe flows, boundary layer flows, and jets. Recent experiments, direct numerical simulations, and Brownian dynamics simulations have revealed that the stretching of polymer molecules by turbulent flows is the dominant effect lying at the heart of this extraordinary phenomenon [1-6]. Yet an understanding of the mechanism of drag reduction remains obscure due to the complexity of turbulence compounded by the complexity of the constitutive equations describing polymer fluids. Although the ultimate aim is to understand polymer drag reduction in well-developed large Reynolds number wall-bounded turbulence $[7,8]$, we focus here on how polymers affect turbulence near its onset. In this Letter, we show that the polymer contribution to the biaxial extensional viscosity of the fluid affects the turbulent structures resulting in drag reduction.

In the last decade, new light has been shed on the understanding of polymer drag reduction after the discovery of exact nonlinear solutions of the Navier-Stokes equations in parallel shear flows $[9,10]$. These solutions, either stationary or in the form of traveling waves, appear as a pair of counterrotating streamwise vortices that give rise to a spanwise stratification of the velocity field, called streaks. A Kelvin-Helmholtz instability of the streaks nonlinearly feeds energy back to the streamwise vortices, making the cycle self-sustaining [11]. Though these solutions come into existence at Reynolds numbers below the onset of turbulence, they are believed to play an important role at the onset, and it has been suggested that wall-bounded turbulence is organized around these exact coherent structures [12]. This suggestion has sparked interest in understanding how the presence of polymers influences the exact nonlinear solutions, with the aim of revealing the underlying physics of turbulent drag reduction.
Even though detailed numerical studies on the effect of viscoelasticity on exact coherent structures by Graham and co-workers $[13,14]$ have revealed that polymer molecules destroy the streamwise vortices and, thereby reduce drag, important questions remain unanswered. First, it is unclear which part of the self-sustaining process is affected by the presence of polymers. Moreover, the numerical studies [14] have also demonstrated that the elasticity conferred on the fluid by the polymers changes the critical Reynolds number, $\mathrm{Re}_{\mathrm{cr}}$, at which the exact nonlinear solutions first appear. For a very weakly elastic fluid, these solutions appear at $\mathrm{Re}_{\mathrm{cr}}$ smaller than the Newtonian value, while for a strongly elastic fluid, the corresponding critical Reynolds number is larger than the Newtonian one. This nonmonotonic behavior is as puzzling as is the drag reduction phenomenon itself.

In this Letter we address these questions by developing and analyzing a low-dimensional model that describes the influence of viscoelasticity on the self-sustaining process that maintains the exact coherent structures. Lowdimensional models have served as instructive tools for understanding the transition to Newtonian turbulence in parallel shear flows [15]. The first low-dimensional model for the self-sustaining process was developed by Waleffe [11] and later refined by Moehlis et al. [16]. The latter model, which is dubbed the MFE model, reproduces the main phenomenology of the transition to turbulence in plane Couette flow, gives realistic predictions for the transitional Reynolds number, and qualitatively describes the statistical character of weak turbulence close to the transition [16]. Hence, we incorporate viscoelasticity into the MFE model to study qualitatively the changes in the selfsustaining process caused by polymers. As we will show below, our viscoelastic model clearly identifies the streak instability as the part of the self-sustaining process which is affected most by elasticity, and also relates the shift in the critical Reynolds number to the flow kinematics, thereby 
complementing the numerical work of Graham et al. and recent direct numerical simulations of wall-bounded turbulence of viscoelastic fluids [3-6].

To derive our model, we follow Moehlis et al. [16] and consider the flow of an incompressible viscoelastic fluid between two parallel plates moving in opposite directions, separated by a distance $2 d$, with free-slip boundary conditions at the walls, and a coordinate system in which $x$ is the streamwise direction, $y$ is the wall-normal direction, and $z$ is the spanwise direction. A body force acting along $x$ and varying sinusoidally along $y$ drives the flow. We write all velocities in units of $U_{o}$ (laminar velocity at $y=d / 2$ from the top plate), lengths in units of $d$, time in units of $d / U_{o}$, stress in units of $\eta U_{o} / d$, and pressure in units of $\rho U_{o}^{2}$, where $\rho$ is the density and $\eta$ is the viscosity of the fluid. With this scaling, the conservation of mass and momentum give,

$$
\begin{gathered}
\boldsymbol{\nabla} \cdot \mathbf{u}=0 \\
\frac{\partial \mathbf{u}}{\partial t}+\mathbf{u} \cdot \boldsymbol{\nabla} \mathbf{u}=-\boldsymbol{\nabla} p+\frac{1}{\operatorname{Re}} \Delta \mathbf{u}+\frac{1-\beta}{\operatorname{Re}} \boldsymbol{\nabla} \cdot \mathcal{T}+\mathbf{F},
\end{gathered}
$$

where $\operatorname{Re}=U_{o} h / \nu$ is the Reynolds number, $\nu=\nu_{s}+\nu_{p}$ is the total kinematic viscosity of the fluid (sum of solvent and polymer contributions), $\beta=\nu_{s} / \nu$ is the viscosity ratio, $\mathbf{F}$ is the body force that drives the flow, and $\mathcal{T}$ is the polymeric contribution to the stress tensor. In the absence of polymers $(\beta=1)$, the MFE model is derived by approximating the velocity field by

$$
\mathbf{u}(\mathbf{x}, t)=\sum_{i=1}^{9} a_{i}(t) \mathbf{u}_{i}(\mathbf{x}),
$$

where the $a_{i}(t)$ are unknown time-dependent amplitudes, and $\mathbf{u}_{i}$ are known orthonormal divergence-free Galerkin spatial modes. Each $\mathbf{u}_{i}$ represents a particular element of the self-sustaining process: $\mathbf{u}_{1}$ and $\mathbf{u}_{9}$ are the mean-shear modes, $\mathbf{u}_{2}$ and $\mathbf{u}_{3}$ are streaks and streamwise vortices, respectively, and $\mathbf{u}_{4}-\mathbf{u}_{8}$ represent the instability of streaks (see [16] for details). The modes $\mathbf{u}_{i}$ depend on two wave numbers $\alpha$ and $\gamma$ that set the size of the coherent structures in the streamwise and spanwise directions, respectively. Substituting (3) into (2) and projecting the resulting equations on the individual modes in (3), we obtain a system of 9 nonlinear ordinary differential equations for the amplitudes of the modes

$$
\frac{d a_{i}(t)}{d t}=-\kappa_{i} a_{i}(t)+\sum_{j, k=1}^{9} c_{i j k} a_{j}(t) a_{k}(t)+\kappa_{i} \delta_{i 1},
$$

where the last term represents the body force, and the exact form of $c_{i j k}$ is given in Ref. [16].

With polymers present $(\beta<1)$, in addition one needs to specify a relation between the polymeric stress $\mathcal{T}$ and the velocity field $\mathbf{u}$. In this Letter, we focus on the so-called retarded-motion expansion of an Oldroyd-B fluid [17,18], while the results for other rheological models will be reported elsewhere. We approximate the polymeric contri- bution to the stress tensor by a power series in the Weissenberg number $\mathrm{Wi}=\lambda U_{o} / h$ ( $\lambda$ being the relaxation time of polymers) which measures the ability of the flow to stretch the polymers,

$$
\mathcal{T}=-\mathrm{WiD}_{(2)}+\mathrm{Wi}^{2} \mathbf{D}_{(3)}+\mathrm{Wi}^{3} \mathbf{D}_{(4)}+\cdots,
$$

where $\mathbf{D}_{(1)}=\frac{1}{2}\left(\boldsymbol{\nabla} \mathbf{u}+\boldsymbol{\nabla} \mathbf{u}^{T}\right)$ is the rate of deformation tensor, and $\mathbf{D}_{(j+1)}=\partial \mathbf{D}_{(j)} / \partial t+\mathbf{u} . \boldsymbol{\nabla} \mathbf{D}_{(j)}-\mathbf{D}_{(j)} \cdot \boldsymbol{\nabla} \mathbf{u}-$ $\boldsymbol{\nabla} \mathbf{u}^{T} \cdot \mathbf{D}_{(j)}$ are its upper-convected Maxwell derivatives. In rheological literature, the fluids described by (5) are called the second-order fluid, the third-order fluid, etc., depending on the highest power of the strain rate tensor included. A distinct advantage of our approach is that (5) obviates the need for separately specifying the spatial modes for $\mathcal{T}$ similar to (3). While this maintains the dimensionality of the model at the same level as Newtonian, it is noteworthy that the stress tensor effectively contains higher order spatial modes than the velocity field $\mathbf{u}$.

We proceed by assuming that at the level of our lowdimensional model, the presence of polymers does not affect the spatial structure of the velocity field (3), but rather changes the balance between different elements of the self-sustaining process. The Galerkin projection of (2) and (5) on the modes $\mathbf{u}_{i}$ from (3) gives the viscoelastic extension of the MFE model up to the third-order fluid,

$$
\begin{aligned}
\frac{d a_{i}(t)}{d t}= & -\kappa_{i} a_{i}(t)+\sum_{j, k=1}^{9}\left(c_{i j k}+d_{i j k}\right) a_{j}(t) a_{k}(t) \\
& +\sum_{j, k, l=1}^{9} e_{i j k l} a_{j}(t) a_{k}(t) a_{l}(t)+\kappa_{i} \delta_{i 1},
\end{aligned}
$$

where $d_{i j k}$, and $e_{i j k l}$ depend on $\alpha, \gamma, \beta$, Re, and Wi. The coefficients in (6) can be found in [19]. This model reduces to the MFE model in the limit of $\mathrm{Wi} \rightarrow 0$.

In the Newtonian case, the model (4) has steady-state solutions that are low-dimensional approximations to the nonlinear traveling-wave solutions found in plane Couette flow [10]. For $\alpha=1 / 2$ and $\gamma=1$, steady solutions have been shown to first appear at the critical Reynolds number $\operatorname{Re}=308.2$ [16]. Using the same values for the wave numbers, we track steady solutions of the viscoelastic model (6) and, in particular, study how the critical Reynolds number at which the nonlinear solutions first appear changes with increase in the Weissenberg number.

In Fig. 1, we plot the critical Reynolds number $\operatorname{Re}_{\text {cr }}$ as a function of Wi, and observe that for a second-order fluid $\mathrm{Re}_{\mathrm{cr}}$ monotonically decreases with Wi, whereas for a thirdorder fluid, $\mathrm{Re}_{\mathrm{cr}}$ first decreases and then increases with Wi beyond $\mathrm{Wi} \sim 1$. The fourth-order fluid shows trends similar to the third-order fluid, demonstrating that (5) is converging for the range of $\mathrm{Wi}$ of our interest. Figure 1 also clarifies that to understand the dominant effects of the decrease and then increase in $\mathrm{Re}_{\mathrm{cr}}$ with $\mathrm{Wi}$, as found from direct numerical simulations of traveling-wave solutions of a FENE-P fluid and reported in Fig. 4 of [14], it 


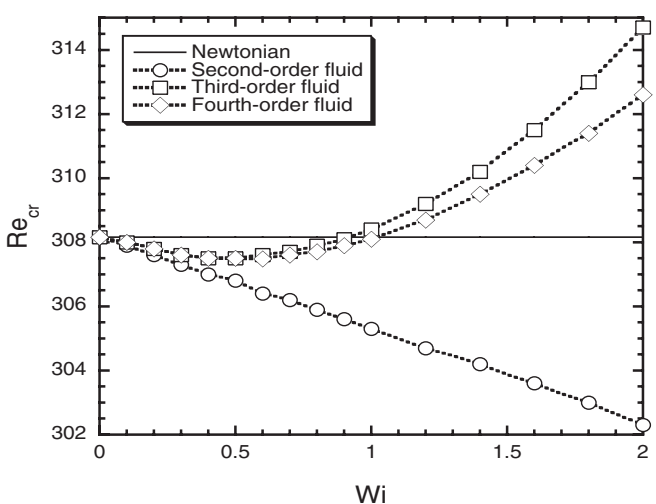

FIG. 1. Steady-state solutions of the viscoelastic 9 dimensional model appear at a critical $\mathrm{Re}_{\mathrm{cr}}$ above or below the Newtonian value (shown by a solid line) depending on the $\mathrm{Wi}$ and the fluid "order".

suffices to study the second- and third-order fluids only. We now proceed to explain the reasons behind this remarkable behavior.

The first clue for the nonmonotonic behavior in Fig. 1 comes from the rheology of Oldroyd-B fluid in extensional flows. In biaxial extensional flow, $v=(\dot{\epsilon} x, \dot{\epsilon} y,-2 \dot{\epsilon} z)$, the biaxial extensional viscosity $\eta_{b}$ initially decreases and then increases as a function of $\lambda \dot{\epsilon}$ as is clear from Fig. 2. This is because the polymer molecules in biaxial extensional flow are stretched in the $x y$ plane at a rate $\dot{\epsilon}$, and compressed along the $z$ direction at double the rate, $2 \dot{\epsilon}$. For small $\lambda \dot{\epsilon}$, compression in the $z$ direction is the largest deformation that the molecules undergo, with much weaker extension in the $x y$ plane. As a result, viscous dissipation is reduced compared to the limit $\dot{\epsilon} \rightarrow 0$, and $\eta_{b}$ decreases with $\lambda \dot{\epsilon}$, a trend followed by the second-order fluid. At larger $\lambda \dot{\epsilon}$, the molecules are extended in the $x y$ plane far more than they are compressed in the $z$ direction, resulting in larger drag from the surrounding flow and $\eta_{b}$ increases with $\lambda \dot{\epsilon}-$ a behavior captured by the third-order fluid. On the other hand, the uniaxial extensional viscosity only monotonically increases because in uniaxial exten-

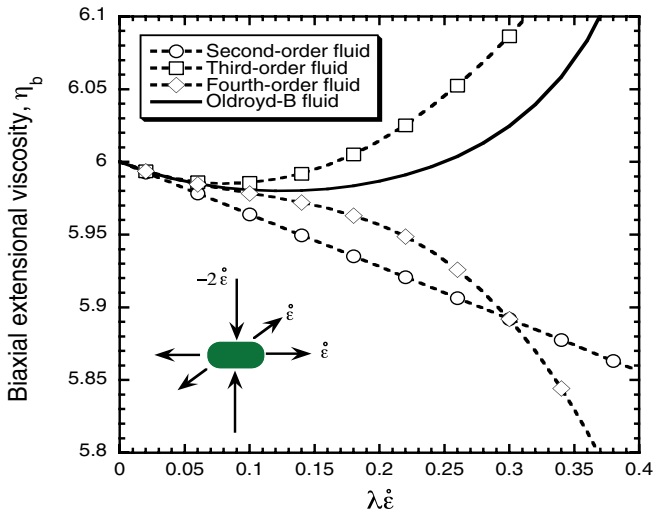

FIG. 2 (color online). Rheology of ordered fluids in biaxial extensional flow, schematically depicted at bottom left. $\eta_{b}$ is made dimensionless with $\eta_{0}$, the zero-shear viscosity. sional flow, the polymers are extended more than they are compressed at any $\lambda \dot{\epsilon}$, resulting in higher viscous dissipation. We observe that the trend of $\eta_{b}$ closely follows the trend of decrease and increase of $\mathrm{Re}_{\mathrm{cr}}$, suggesting that the biaxial extensional viscosity is a key rheological property governing the behavior shown in Fig. 1, in tune with the conclusions of Terrapon et al. [6], who recognized the relevance of polymers stretching in regions of biaxial extension to turbulent drag reduction.

Our observations motivate a search for regions of biaxial and uniaxial extension in the flow field of our model. To characterize biaxial extension, we consider the sign of one of the invariants of the rate of deformation tensor $R=$ $-\operatorname{det}(\mathbf{D})$ which determines the type of flow: for uniaxial extension $R<0$, for biaxial extension $R>0$, and for simple shear flow $R=0$. In Fig. 3(a), the region of strongest biaxial extension is present in the streaks between the streamwise vortices. Polymer molecules have been shown to extend in the streaks in [20], and hence it is no surprise to find a region of relatively strong extension in the region of the streaks. What is surprising, however, is that most of the extension in the streaks arises from biaxial extension, in particular, as can be seen in Fig. 3(a). Moreover, these regions of significant biaxial extension are produced by the coupling of the modes corresponding to the linear instability of the streaks $\left(a_{6}, a_{7}\right.$, and $\left.a_{8}\right)$, also called wallnormal vortex modes, and, most counterintuitively, the modes corresponding to mean shear $\left(a_{1}\right.$ and $\left.a_{9}\right)$. The rest of the modes contribute to uniaxial extension in the flow, which is much weaker than the biaxial extension flow produced by the instability modes.

Another clue for understanding the effects of elasticity on the self-sustaining process comes from analyzing the dynamical system, (6). We find that of the 182 elastic terms present in (6), only 11 terms are responsible for the change in $\mathrm{Re}_{\mathrm{cr}}$ presented in Fig. 1. These dominant elastic terms of the second- and third-order fluids appear only in the equations for the instability modes $a_{6}$ and $a_{7}$ :

$$
\begin{aligned}
\dot{a}_{6}= & \text { N.t. }+\ldots a_{1} a_{7}+\ldots a_{8} a_{9}, \\
\dot{a}_{7}= & \text { N.t. }+\ldots a_{6}\left(a_{1}+a_{9}\right)+\ldots a_{8}\left(a_{1}^{2}+a_{9}^{2}+a_{1} a_{9}\right) \\
& +\ldots a_{7} a_{9}\left(a_{1}+a_{9}\right)+\ldots a_{7}^{2}\left(a_{6}+a_{8}\right),
\end{aligned}
$$

where "N.t." denotes Newtonian terms and where we have omitted numerical coefficients. Equation (4) with (7) replacing the equations for $\dot{a}_{6}$ and $\dot{a}_{7}$ is the "most minimal model" needed to explain the changes in $\mathrm{Re}_{\mathrm{cr}}$ shown in Fig. 1. We also observe that nearly every dominant term in (7) consists of interactions between the mean shear, $a_{1}$ or $a_{9}$, and one of the instability modes, $a_{6}, a_{7}$, and $a_{8}$. Hence, not only do the instability modes produce regions of strong biaxial extension in the flow, but elasticity acts on the selfsustaining process through the interactions between them.

Finally, to understand how the trend in biaxial extensional viscosity in Fig. 2 can explain Fig. 1, we examine the elastic polymer force arising in (2) due to (5), given by $\mathbf{f}=$ $\frac{2(1-\beta)}{\operatorname{Re}} \boldsymbol{\nabla} \cdot \mathcal{T}$. In Figs. 3(b) and 3(c), we show the vectors of 


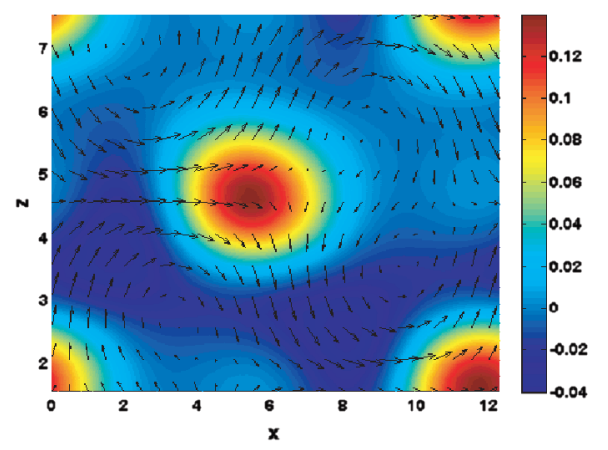

(a)

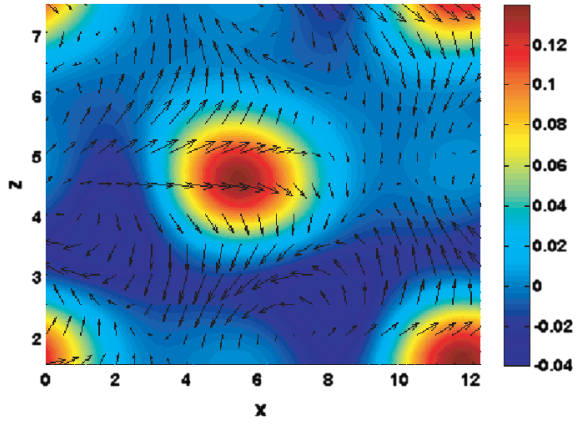

(b)

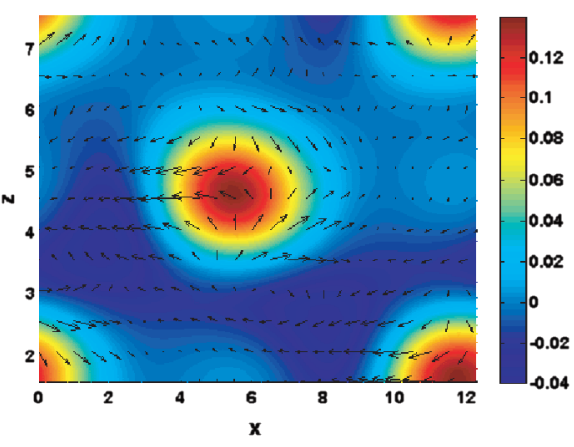

(c)

FIG. 3 (color online). At $\operatorname{Re}=350$ for $x \in[0,4 \pi], z \in[\pi / 2,5 \pi / 2]$ in the $x-z$ plane at $y=0.62$, the arrows representing the $x$ and $z$ components of velocity in (a) and polymer force in (b) and (c). The background color map shows regions of biaxial (red) extension seen as bright halos in the center and corners of the figures, and regions of uniaxial extension (blue) interspersed between them.

elastic forces that are meant to be viewed along with Fig. 3(a). We find that the elastic forces of the secondorder fluid in Fig. 3(b) assist flow in regions of strong biaxial extension and resist flow in regions of uniaxial extension. On the other hand, the third-order fluid elastic forces in Fig. 3(c) act to resist flow both in regions of uniaxial and biaxial extension. Both these observations are consistent with Fig. 2 as well as the trends in uniaxial extensional viscosity discussed earlier.

Based on our observations, we propose a mechanism by which polymers affect the existence and nature of the exact coherent structures shown in Fig. 1. Weak elasticity, as in the second-order fluid, enhances the linear instability of the streaks by assisting flow in the dominant biaxial extensional flow region between the streaks, as is evident from Fig. 3(b). Consequently, the counterrotating streamwise vortices that are sustained by the instability of the streaks become stronger, giving rise to even stronger stratification in the spanwise direction (streaks), and thereby strengthening the self-sustaining process. Stronger elasticity, as in the third-order fluid, suppresses the instability of the streaks, since elasticity now opposes flow in both biaxial and uniaxial extensional flow regions, as we show in Fig. 3(c). This results in the rolls and streaks becoming weaker and a weaker self-sustaining process. Since less mean momentum is now advected away from the walls, the mean shear increases and drag is reduced.

Our low-dimensional model not only reveals the lowestorder effects of elasticity on the coherent structures typical of wall-bounded turbulence, but is also the first step of a systematic explanation of higher order effects that can be captured by adding higher order terms either to the amplitude Eq. (6) or the retarded-motion expansion (5), or both. Since the retarded-motion expansion is general, effects such as shear-thinning, normal stress differences, or elastic and viscous effects characteristic of fibers, platelets, surfactant solutions, etc., can also be considered within the same framework.

A.M. acknowledges financial support from NWO(GBE). A. R. thanks the Rackham Graduate School for financial support, and FOM for sponsoring his visit to
Leiden.

*Electronic address: rlarson@umich.edu

[1] J. L. Lumley, Annu. Rev. Fluid Mech. 1, 367 (1969).

[2] P. S. Virk, AIChE J. 21, 625 (1975).

[3] K. D. Housiadas and A. N. Beris, Phys. Fluids 15, 2369 (2003).

[4] T. Min, J. Yoo, H. Choi, and D. D. Joseph, J. Fluid Mech. 486, 213 (2003).

[5] P. K. Ptasinsky, B. J. Boersma, F. T. M. Nieuwstadt, M. A. Hulsen, B. H. A. A. V. den Brule, and J. C. R. Hunt, J. Fluid Mech. 490, 251 (2003).

[6] V.E. Terrapon, Y. Dubief, P. Moin, E. S. G. Shaqfeh, and S. K. Lele, J. Fluid Mech. 504, 61 (2004).

[7] R. Benzi, E. D. Angelis, V.S. L'vov, and I. Procaccia, Phys. Rev. Lett. 95, 194502 (2005).

[8] A. Roy and R. G. Larson, Applied Rheology 15, 370 (2005).

[9] B. Hof, C. W. H. van Doorne, J. Westerweel, F. T. M. Nieuwstadt, H. Faisst, B. Eckhardt, H. Wedin, R. R. Kerswell, and F. Waleffe, Science 305, 1594 (2004).

[10] F. Waleffe, Phys. Rev. Lett. 81, 4140 (1998).

[11] F. Waleffe, Phys. Fluids 9, 883 (1997).

[12] B. Hof, C. W. H. van Doorne, J. Westerweel, and F. T. M. Nieuwstadt, Phys. Rev. Lett. 95, 214502 (2005).

[13] P. A. Stone, F. Waleffe, and M. D. Graham, Phys. Rev. Lett. 89, 208301 (2002).

[14] P. A. Stone, A. Roy, R. G. Larson, F. Waleffe, and M. D. Graham, Phys. Fluids 16, 3470 (2004).

[15] J. S. Baggett and L. N. Trefethen, Phys. Fluids 9, 1043 (1997).

[16] J. Moehlis, H. Faisst, and B. Eckhardt, New J. Phys. 6, 56 (2004).

[17] R. G. Larson, Constitutive Equations for Polymer Melts and Solutions (Butterworth, Massachusetts, 1988).

[18] R. B. Bird, C. F. Curtiss, R. Armstrong, and O. Hassager, Dynamics of Polymeric Liquids (John Wiley \& Sons, Inc., New York, 1987), Vol. 1.

[19] A. Roy, Ph.D. dissertation, University of Michigan, Ann Arbor, 2006.

[20] P. A. Stone and M.D. Graham, Phys. Fluids 15, 1247 (2003). 